\title{
Suppression of membrane vesiculation as anticoagulant and anti-metastatic mechanism. Role of stability of narrow necks
}

\author{
Roman Štukelj ${ }^{1}$, Vid Šuštar ${ }^{2}$, Anita Mrvar-Brečko ${ }^{3}$, Peter Veranič̌ ${ }^{4}$,Henry Hägerstrand ${ }^{5}$, \\ Veronika Kralj-Iglič ${ }^{1}$ and France Sevšek ${ }^{1}$ \\ ${ }^{1}$ Laboratory of Clinical Biophysics, Faculty of Health Sciences, University of Ljubljana, Ljubljana, Slovenia \\ ${ }^{2}$ Laboratory of Clinical Biophysics, Chair of Orthopaedic Surgery, Faculty of Medicine, University of Ljubljana, Ljubljana, Slovenia \\ ${ }^{3}$ Clinical Department for Anaesthesiology and Intensive Care of Operative Branches, Ljubljana University Medical Centre, \\ Ljubljana, Slovenia \\ ${ }^{4}$ Institute of Cell Biology, Faculty of Medicine, University of Ljubljana, Ljubljana, Slovenia \\ ${ }^{5}$ Department of Biology, Åo Akademi University, Åbo/Turku, Finland
}

\begin{abstract}
Nanovesicles that are pinched off from biological membranes in the final stage of budding constitute a cell-cell communication system. Recent studies indicate that in vivo they are involved in blood clot formation and in cancer progression. The bud is connected to the mother membrane by a thin neck so it dwells close to the mother membrane. Using the electron microscopy we have observed in blood cells that adhesion between the membrane of the bud and of the mother cell in the vicinity of the neck took place and prevented the bud to pinch off from the mother vesicle. The same effect was observed in giant phospholipid vesicles (GPVs) due to attractive interaction between the bud and the mother vesicle mediated by the plasma protein beta-2-glycoprotein I. The stability of the neck is important for this process. By using Fourier method we analyzed thermal fluctuations of a GPV while a protrusion composed of beads connected by thin necks was spontaneously integrated into the mother GPV. Stepwise change of Fourier coefficients indicates an increased stability of necks which contributes to the retention of buds by the mother membrane and promotes anticoagulant and anti-metastatic mechanism by suppression of nanovesiculation.
\end{abstract}

Key words: Flickering - Microvesicles - Nanovesicles - Thrombosis - Cancer

\section{Introduction}

Biophysical properties of biological membranes influence vital mechanisms taking place in cells. Hitherto, attention has been devoted mostly to the mildly curved membranous structures such as plasma membrane. Recently, another membranous pool - spontaneously stable membranous nanostructures - has been recognized in cells (Hägerstrand and Isomaa 1989, 1992; Rustom et al. 2004; Schara et al. 2009; Kralj-Iglič 2012) and also in phospholipid systems (Mathivet et al. 1996; KraljIglič et al. 2001; Iglič et al. 2003; Mareš et al. 2008; Rappolt et al. 2008). Such structures are for example buds (Kralj-Iglič et

Correspondence to: Veronika Kralj-Iglič, Laboratory of Clinical Biophysics, Faculty of Health Sciences, University of Ljubljana, Zdravstvena 5, SI-1000 Ljubljana, Slovenia

E-mail: veronika.kralj-iglic@fe.uni-lj.si al. 2000, 2002), nanotubules (Kralj-Iglič et al. 2002, Iglič et al. 2003; Rustom et al. 2004; Galkina et al. 2005; Gimsa et al. 2007; Veranič et al. 2008), nanovesicles (Hägerstrand and Isomaa 1989, 1992; Ratajczak 2006; Junkar et al. 2009; Mrvar-Brečko et al. 2010; Šuštar et al. 2011a, b) and narrow necks (Kralj-Iglič et al. 2006; Iglič et al. 2007; Jorgačevski et al. 2010).

Membranous nanostructures are important for the function of cells and tissues since they constitute cell-cell communication system. Signalling by nanovesicles (NVs) may contribute to a variety of biological processes e.g. spreading of inflammation (Distler et al. 2005; Pisetsky 2009), transport of infectious particles (Fevrier et al. 2004; Robertson et al. 2006; Vella et al. 2008) and progression of tumour in cancer (Yu et al. 2005; Janowska-Wieczorek et al. 2006; Ratajczak et al. 2006; $\mathrm{Al}$ Nedawi et al. 2008). Cancer cell NVs-borne oncoproteins, lipids, and nucleic acids (DNA, mRNA, microRNA) may be transferred to other cells and thereby affect tumor progression, 
immunotolerance, invasion, angiogenesis, and metastasis (Rak 2010), while cancer cell NVs-borne tissue factor is involved in coagulopathy leading to an increased risk for clot formation in blood vessels (Müller et al. 2000). As the same NVs may carry molecules that are involved both in cancer progression and in thromboembolic disorders, it was suggested that they could play an important role in coagulopathies in cancer described as the Trousseau syndrome (Šuštar et al. 2009). However, knowledge on the underlying mechanisms is rudimentary, which is one of the obstacles that targeted manipulation of nanovesiculation is not yet established as a standardized method in treatment of respective diseases.

Clinical studies have shown that concentration of NVs in isolates from blood of patients with cancer and with thromboembolic disorders was higher than in isolates from blood of healthy subjects (Dignat-George et al. 2004; Janša et al. 2008). It was therefore suggested (Urbanija et al. 2007; Frank et al. 2008) that suppression of nanovesiculation could imply a possible anti-metastatic and anticoagulant mechanism. Since NVs are very small their morphology could not be observed live by optical microscope. However, giant phospholipid vesicles (GPVs) are large enough while some features in GPVs are the same as in NVs. Therefore unilamellar GPVs (Bagatolli et al. 2000; Peterlin and Arrigler 2008; Pavlič et al. 2010; Šuštar et al. 2012) are a convenient system to study the membrane features such as budding (Käs and Sackmann 1991) and thermal fluctuations of the shape (Bivas et al. 1987, 1992, 2003, 2010; Faucon et al. 1989; Méléard et al. 1997; Vitkova et al. 2003; Genova et al. 2009).

It was observed in concentrated suspensions of GPVs that added substances (in particular, plasma protein beta-2glycoprotein I and antiphospholipid antibodies dissolved in phosphate buffer saline) may cause adhesion between membranes; adhesion took place even when both membranes were negatively charged (Ambrožič et al. 2006). In a budding vesicle, the added beta-2-glycoprotein I caused attraction of the bud to the mother membrane and its adhesion to the membrane (Urbanija et al. 2007; Frank et al. 2008). It was interpreted that added substances may mediate attractive interaction between membranes (Urbanija et al. 2007). A model describing the adhesion due to the presence of mediating molecules was proposed, suggesting that the mediated attraction derives from a decrease of the free energy of the system due to orientational ordering of mediating molecules with spatially distributed charge (Urbanija et al. 2008a,b). The interaction turned out to be short-ranged (Urbanija et al. 2008b); adhesion will likely take place if the distance between membranes is smaller than few nanometers. For a bud, these conditions are fulfilled when it is connected to the mother vesicle by a short and thin but stable neck. Adhesion of a bud to the mother membrane was indeed observed in GPVs (Urbanija et al. 2007), but to our best knowledge, it has not yet been reported in cells. Here we present evidence that a similar process takes place also in blood cells.
Theoretically it was predicted that shapes in which a bud is connected to the mother membrane with a thin neck correspond to the global minimum of the membrane free energy and are therefore stable (Kralj-Iglič et al. 2006). To validate these predictions, we studied the stability of shapes with thin necks experimentally. In GPVs and in cells, tube-like protrusions are commonly observed. These protrusions may also be undulated thereby forming multiple necks by which the "beads" are connected to form the protrusion (Kralj-Iglič et al. 2001; Iglič et al. 2007). Such shapes are observed in the last stage of spontaneous process in which myelin-like protrusions are integrated into the mother vesicle (Fig. 1); when GPVs are formed by electroformation they are connected by a network of nanotubules (Mathivet et al. 1996; Kralj-Iglič et al. 2001). Rinsing GPVs from electroformation chamber tears this network, however, its remnants remain attached to GPVs. Due to a spontaneous process the average mean curvature of the GPVs decreases with time (Kralj-Iglič et al. 2001; Božič et al. 2002). The reason for the transformation is not known, but presumably, phospholipid molecules are slowly removed from the outer membrane layer due to equalization of the chemical potential in the solution and in the membrane, degradation of lipid and lipid flip flop (Kralj-Iglič et al. 2001). The protrusion (Fig. 1A) shortens and thickens (Fig. 1B-D) and eventually exhibits a bead-like bud (Fig. 1E) which further transforms by diminishing the number of beads (Fig. 1E-I). Finally, the neck, connecting the protrusion to the mother vesicle opens and the protrusion is integrated into the mother vesicle. To study the effect of the neck formation on the stability of the shape, we observed thermal fluctuations of the mother GPV shape during this process.

We present evidence in favour of the hypothesis that mediated interaction between membranes may suppress the nanovesiculation process in cells by causing adhesion of a bud to the mother membrane. We show below that this process can take place in blood cells. Further, we focus on the feature that enables such effect, i.e. stability of a narrow neck connecting mother and daughter vesicles by quantitatively determining how the fluctuations of the globular part develop in the process where the myelin-like protrusion is slowly integrated into the mother globule.

\section{Materials and Methods}

\section{Blood sampling}

Blood was collected from authors into $2.7 \mathrm{ml}$ tubes containing $270 \mu \mathrm{l}$ trisodium citrate at a concentration $109 \mathrm{mM}$. Evacuated tubes (BD Vacutainers, Becton Dickinson, CA) and 21-gauge needles (length $70 \mathrm{~mm}$, inner radius $0.4 \mathrm{~mm}$ ) (Microlance, Becton Dickinson, NJ, USA) were used for blood sampling. Prior to sampling the tubes were incubated 

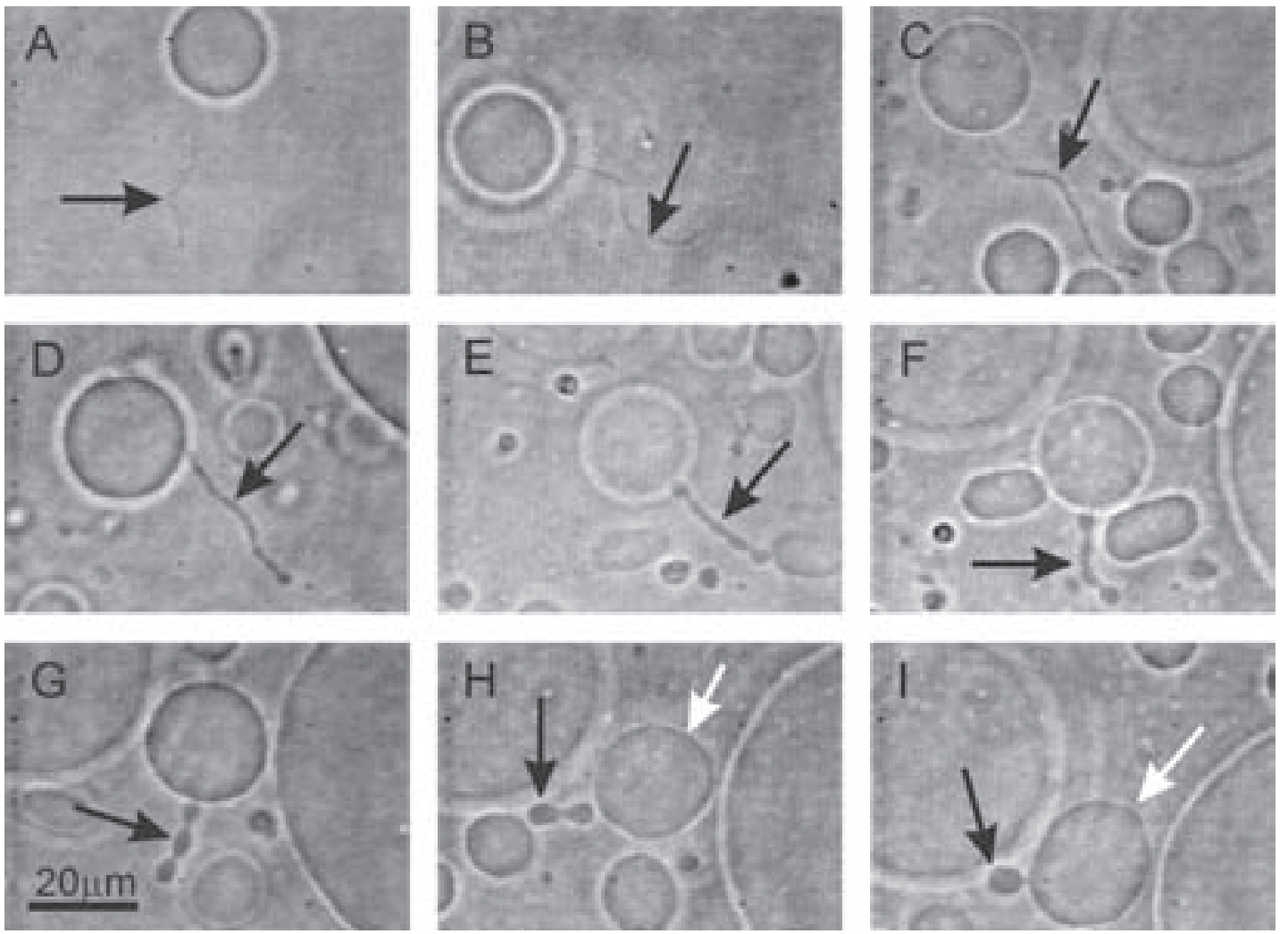

Figure 1. Time course of the spontaneous slow shortening of the myelin protrusion of a giant phospholipid vesicle made of POPC in sugar solution (A-I). Black arrows point to the protrusion while white arrows point to the mother vesicle.

in a rotating temperature-regulated centrifuge at $37^{\circ} \mathrm{C}$ and kept at this temperature in a thermo-block (Domel d.o.o., Železniki, Slovenia) during the handling of samples.

\section{Induction of nanovesiculation by calcium ionophore A21387}

After blood collection, blood was centrifuged at $1000 \times g$, $10 \mathrm{~min}$, at room temperature. Supernatant blood plasma and thrombocyte-leukocyte fraction (buffy coat) were removed by pipetting, replaced with equal volume of Allen's buffer (145 mM NaCl, $5 \mathrm{mM} \mathrm{KCl,} 1 \mathrm{mM} \mathrm{Na} \mathrm{HPO}_{4}, 10$ mM glucose, $1 \mathrm{mM} \mathrm{MgSO}_{4} \cdot 7 \mathrm{H}_{2} \mathrm{O}, 1 \mathrm{mM} \mathrm{CaCl}_{2} \cdot 2 \mathrm{H}_{2} \mathrm{O}$, used throughout the procedure) and gently mixed. The centrifugation and buffer exchange was performed 3 times. Erythrocytes were buffer-diluted approximately 1:10 v/v to a final concentration of $1.65 \times 10^{8}$ cells $/ \mathrm{ml}$. Buffer-washed erythrocytes were added to $2 \mu \mathrm{M}$ calcium ionophore A21387 (Calbiochem-Behring Corp., La Jolla, CA, USA) dissolved in buffer. Aliquotes of the suspension, pre-warmed to $37^{\circ} \mathrm{C}$ were pipetted into $2 \mathrm{ml}$ epruvettes and incubated for $15 \mathrm{~min}$ at this temperature. For fixation, a 5\% buffer solution of formaldehyde (FD) or/and of $0.01 \%$ glutaraldehyde (GA), was added and incubated for $1 \mathrm{~h}$ at room temperature. Samples were washed 3 times by exchanging $500 \mu$ l of buffer after centrifugation at $1000 \times g$, for $1 \mathrm{~min}$, at room temperature.

\section{Isolation of $\mathrm{NVs}$}

Centrifugation of aliquoted samples started within $20 \mathrm{~min}$ after the acquisition of blood. In order to separate cells from plasma, blood was centrifuged at $1550 \times g$ for $20 \mathrm{~min}$ in a centrifuge Centric 400/R (Domel d.o.o., Železniki, Slovenia). The upper $250 \mu \mathrm{l}$ of plasma was slowly removed from each tube and placed in a $1.5 \mathrm{ml}$ Eppendorf tube. The samples were centrifuged at $17570 \times g$ for $30 \mathrm{~min}$ in a centrifuge Centric 200/R (Domel d.o.o., Železniki, Slovenia). The supernatant $(225 \mu \mathrm{l})$ was discarded and the pellet $(25 \mu \mathrm{l}$ resuspended in $225 \mu$ l of citrated phosphate buffer saline (PBS). Samples were centrifuged again at $17570 \times g$ for $30 \mathrm{~min}$, the supernatant $(225 \mu \mathrm{l})$ discarded and the pellet $(25 \mu \mathrm{l})$ re-suspended in appropriate quantity of citrated PBS.

\section{Scanning electron microscopy (SEM)}

The NVs were suspension-fixed in $1 \%$ glutaraldehyde dissolved in citrated PBS for $60 \mathrm{~min}$ at $22^{\circ} \mathrm{C}$, post-fixed for 
60 min at $22^{\circ} \mathrm{C}$ in $1 \% \mathrm{OsO}_{4}$ dissolved in $0.9 \% \mathrm{NaCl}$, and then dehydrated in a graded series of acetone/water $(50-100 \%$, $\mathrm{v} / \mathrm{v})$. The samples were critical-point dried, gold-sputtered, and examined using a LEO Gemini 1530 (LEO, Oberkochen, Germany) scanning electron microscope.

\section{Transmission electron microscopy (TEM)}

Samples were fixed in $2.5 \%$ glutaraldehyde and post-fixed in $1 \% \mathrm{OsO}_{4}$. After dehydration they were embedded in epon. Ultrathin sections were counterstained with uranyl acetate and lead citrate. Samples were examined with transmission electron microscope $100 \mathrm{CX}$ (Jeol, Japan) at working voltage $80 \mathrm{kV}$.

\section{Preparation of giant phospholipid vesicles}

The phospholipid 1-palmitoyl-2-oleoyl-sn-glycero-3phosphocholine (POPC) was purchased from Avanti Polar Lipids. The vesicles were prepared by the modified method of electroformation (Angelova et al. 1992). The electroformation of GPVs was performed at room temperature. In the procedure, $20 \mu \mathrm{l}$ of phospholipid dissolved in 2:1 chloroform/methanol mixture, was spread over a pair of platinum electrodes. The solvent was allowed to evaporate for two hours. The electrodes were placed into the electroformation chamber which was then filled with $2 \mathrm{ml}$ of $0.2 \mathrm{M}$ sucrose solution. An alternating electric field was applied as described in Kralj-Iglič et al. (2001). After the electroformation the content of the chamber was poured out into a plastic beaker. Then, the chamber was rinsed with $2 \mathrm{ml}$ of $0.2 \mathrm{M}$ solution of sugar of different molecular weight (glucose) and the contents of the chamber were added to the solution that was already in the plastic beaker. The solution was gently mixed. We used an equiosmolar solution of lighter sugar (glucose) for rinsing the vesicles into the observation chamber so that the vesicles containing the solution of heavier sugar (sucrose) sunk to the bottom of the observation chamber and made the observation easier.

For determination of shape fluctuations, the solution containing the vesicles was immediately after the formation placed into the observation chamber made by a pair of cover glasses and sealed by vacuum grease. The vesicles were observed by the inverted microscope Zeiss IM 35 with the phase contrast optics. The microscope was focused on the globular part therefore the protrusion could not be clearly seen for most of the time. Video camera Sony PCD-IRIS (model SSC-M370CE) attached to the microscope served to record the images. The frame grabber (Data Translation DT2851) in a personal computer was used to digitize the images at the rate of about one per second. This resulted in a series of 1600 pictures of the same vesicle. The pictures size was $512 \times 512$ pixels with 256 gray levels.
For mediated interaction between membranes GPVs were left to sediment in the gravitation field for 12 hours. Beta-2-glycoprotein I (Hyphen BioMed, France) dissolved in PBS was added to the suspension at a final concentration of $100 \mathrm{mg} / \mathrm{l}$.

\section{Image Processing}

Shading or intensity inhomogeneity is an adverse phenomenon in microscopy manifesting itself as smooth intensity variations not present in the original (Likar et al. 2000; Russ 2000) and may arise from imperfections in the image acquisition process. Therefore, correction is needed after image acquisition. We suppressed the shading of an acquired image $(N)$ by using an additive $\left(S_{\mathrm{A}}\right)$ and a multiplicative $\left(S_{\mathrm{M}}\right)$ shading component to transform the acquired image into the corrected image $(U)$,

$$
U=\frac{N-S_{\mathrm{A}}}{1+S_{\mathrm{M}}}
$$

The shading components $S_{\mathrm{A}}$ and $S_{\mathrm{M}}$ were approximated by a globally neutralized second order polynomial $S$

$$
S=s_{x} x+s_{y} y+s_{x y} x y+s_{x x}\left(x^{2}-\frac{W^{2}}{12}\right)+s_{y y}\left(y^{2}-\frac{H^{2}}{12}\right)
$$

defined by five parameters: $s_{x}, s_{y}, s_{x x}, s_{x y}, s_{y y} . W$ is the width of the image and $H$ is its height. To suppress the shading, the origin was taken at the centre of the image. The optimal parameters of both components $\mathbf{s}_{\mathrm{A} 0}, \mathbf{s}_{\mathrm{M} 0}$ were found by Powell's multidimensional directional set method and Brent's one-dimensional optimization algorithm (Press et al. 1992) minimizing the entropy $(E)$ of the acquired image,

$$
\left\{\mathbf{s}_{\mathrm{A} 0}, \mathbf{s}_{\mathrm{M} 0}\right\}=\arg \min _{\left\{\mathbf{s}_{\mathrm{A} 0}, \mathbf{s}_{\mathrm{M} 0}\right\}}\left\{E \frac{\left(N-S_{\mathrm{A}}\right)}{1+S_{\mathrm{M}}}\right\}
$$

The entropy $E$ was calculated from the probability distribution which was estimated by normalization of the corresponding intensity histogram of the transformed image $U$. A detailed description of the shading correction method can be found in Likar et al. $(2000,2001)$.

The vesicle outlines were determined from the corrected images (Sevšek and Gomišček 2004; Usenik et al. 2011). The images were binarized and slightly smoothened if necessary. Since in our case the contrast of the binarized image was good, the application of gradient operators (Gonzalez 1992) was not necessary and the contour could be determined directly. From the binary images the vesicle 4-connected contour was determined by tracing the inner boundary of the white halo surrounding the vesicle. The implementation of the contourfollowing routine was based on a maze solving algorithm. The coordinates of the contours were calculated relatively to the contour centres. An example of the processed vesicle image and the obtained contour are shown in Fig. 2. 
Some images (in the interval between 850 and 1000 s) had to be excluded from the analysis due to insufficient quality.

\section{Determination of the vesicle shape}

The shape of a nearly spherical vesicle can be expressed by using the expansion into spherical harmonics

$$
R(\theta, \varphi)=R_{\mathrm{s}}\left(1+\sum_{\ell=0}^{\ell \max } \sum_{m=-\ell}^{m=\ell} u_{\ell m} Y_{\ell m}(\theta, \varphi)\right)
$$

where $R(\theta, \varphi)$ is the distance from the contour centre to the membrane, $R_{\mathrm{S}}$ is the effective radius of the mother globule, $u_{\ell m}$ are the Fourier coefficients and $Y_{\ell m}$ are the normalized spherical harmonics (Abramowitz and Stegun 1970),

$$
Y_{\ell m}(\theta, \varphi)=N_{\ell m}(\theta, \varphi) P_{\ell m}(\cos \theta) \exp (\mathrm{im} \varphi)
$$

$P_{\ell m}(\cos \theta)$ are the associated Legendre functions and $N_{\ell m}(\theta, \varphi)$ are the normalization factors,

$$
N_{\ell m}=\sqrt{\frac{(2 \ell+1)(\ell-|m|)}{4 \pi(\ell+|m|)}}
$$

The effective radius $R_{\mathrm{S}}$ is introduced in such a way that all the Fourier coefficients $u_{\ell m}$ are small. The shape of the cross section of the vesicle is obtained from Eq. (4) by taking $\theta=\pi / 2$,

$$
R(\theta=\pi / 2, \varphi)=R_{\mathrm{s}}\left(1+\sum_{-\ell_{\max }}^{\ell_{\max }} u_{m} \exp (\operatorname{im} \varphi)\right)
$$



Figure 2. The processed image of the phospholipid vesicle and the vesicle contour. The microscope was focused on the globular part (white arrow). A part of the protrusion can be seen at the bottom right (black arrow). White cross marks the centre of the contour. The diameter of the globular part of the vesicle was about $15 \mu \mathrm{m}$.
The corresponding Fourier coefficients are

$$
u_{m}=\sum_{\ell=|m|}^{\ell \max } u_{\ell m} N_{\ell m} P_{\ell m}(0)
$$

where

$$
P_{\ell m}(0)=\frac{2^{m}}{\pi} \cos \left(\frac{\pi}{2}(\ell+m)\right) \frac{\Gamma(\ell / 2+|m| / 2+1 / 2)}{\Gamma(\ell / 2-|m| / 2+1)}
$$

Since in our case the computational time was not crucial we used a straightforward method to determine the Fourier coefficients and to perform the experimentally obtained least square contour fitting by the expression (7). The resulting equations form a linear system that was solved for the Fourier coefficients by decomposing the matrix into the product of the lower and the upper triangular matrices - the method which is known as LU (lower and upper triangular decomposition) method (Press et al. 1992). From the product, solutions were calculated by a simple substitution.

\section{Results}

Fig. 3 shows budding of biological membranes and adhesion of buds to the mother membrane. Adding ionophore to the suspension of erythrocytes caused a discocyte - echinocyte transformation (Fig. 3A). Budding of the membrane took place at the tips of the echinocyte spicules (Fig. 3A). Some buds prolonged into tubular protrusions (Fig. 3A, white arrow) while others appeared as composed of a series of globular units. Fig. 3B shows the adhesion of such units to each other (Fig. 3B, white arrows and black arrow). Adhesion of a bud to the mother membrane, or to the membrane of the adjacent cell, was observed also in platelets (Fig. 3C, white arrows) and in GPVs (Fig. 3D, white arrow) while adhered nanovesicles were found in leukocytes (Fig. 3E) and erythrocytes (Fig. 3F).

Spherical nanovesicles may have adhered to the erythrocyte membrane (Fig. 3F, black arrow) while a contact between two erythrocytes was made through nanovesicles adhering at their tips (Fig. 3F, gray arrow). Transmission electron image shows that the nanostructures were lighter (Fig. 3F) indicating that they do not contain hemoglobin.

Either the membrane of these structures was permeable to hemoglobin while the connection with the mother cells (the necks) did not allow effective exchange of this protein between them and the respective mother cells, or - adhered nanovesicles did not derive from the cell to which they had adhered, but from another cell. As the adhered nanostructures were lighter, the mother cell was most probably not an erythrocyte.

Fig. 4 shows the time dependence of the averaged square of the Fourier coefficients normalized by the square of the effective radius (A) and of the effective radius of the mother vesicle $R_{\mathrm{S}}$ (B) corresponding to the last stages of the slow 
spontaneous shortening of the myelin-like protrusion and its integration with the mother vesicle. The effective radius of the mother vesicle $R_{\mathrm{S}}$ on the average increased (Fig. 4B). However, the increase of $R_{\mathrm{S}}$ was not monotonous. Rather, a peculiar stepwise pattern could be observed. The abrupt increase of the effective radius corresponds to a transformation of the protrusion into the elongated shape with one bead less. The duration of steps increases so that the protrusion with three beads is less persistent than the protrusion with two beads and the latter is less persistent than the protrusion with one bead (Fig. 4B).

The contribution of the Fourier coefficient with $m=2$ is the largest, however, also the coefficients with higher $m$ can be noted (Fig. 4A). On the average all the coefficients increase with time. The increase is especially large at the end of the sequence when the globular shape of the vesicle is reached. At this point the effective radius of the mother globule and the Fourier coefficients abruptly increase (Figs. 4A,B).

\section{Discussion}

Adhesion of the bud to the mother membrane was first observed in GPVs upon addition of a cofactor for binding of antiphospholipid antibodies to negatively charged phospholipids (beta-2-glycoprotein I) dissolved in phosphate buffer saline (Urbanija et al. 2007). Although the vesicle was composed of a mixture of POPC and phosphatidylserine which was at neutral $\mathrm{pH}$ in the solution negatively charged, the bud adhered to the mother vesicle (Urbanija et al. 2007) while in the case of a vesicle with long bead-like protrusion, the beads adhered to each other (Urbanija et al. 2007). This effect was attributed to the mediating effect of the added molecules (beta-2-glycoprotein I). In the control experiment where phosphate buffer saline alone was added to the vesicles, the necks connecting the beads to each other and the protrusion to the mother vesicle were torn to yield small spherical daughter vesicles which were free to move away from the mother vesicle. Tearing of the necks was preceded by vigorous movements of the protrusion (most probably due to concentration gradient caused by adding the sample), so it could be interpreted that the reason for the tearing was mechanical in nature. Adhesion of platelet buds to the mother membrane was suggested as a possible anticoagulant mechanism since platelet NV s constitute a catalytic surface for blood clot formation (Müller et al. 2000), while adhesion of tumour cell buds to the mother membrane was suggested as a possible anti-metastatic mechanism, since NVs are involved in tumour progression (Janowska-Wieczorek et al. 2006). The interaction mediated by beta-2-glycoprotein I could be explained by the structure of beta-2-glycoprotein I exhibiting a hydrophobic domain (Wang et al. 1998) and two positively charged domains (Kertesz et al. 1995; Bouma et al. 1999). It is energetically advantageous that the hydrophobic domain is inserted into one membrane while the positively charged domains interact with the other membrane to form a "bridge" (Urbanija et al. 2007, 2008a). However, as the membrane headgroup interface is a source of electric field (in the case of charged or multipolar headgroups) and screening of this field takes place due to the presence of ions in the adjacent solution, a gradient of electric field is created close to the phospholipid headgroup interface. In the solution, molecules with internally distributed charge will therefore orient in this gradient as to minimize their energy. The decrease of the free energy of the system is the greatest when the two interacting membranes are separated by a small distance within which the mediating molecules are orientationally ordered (Urbanija et al. 2008b). The attractive interaction would take place if the free energy minimum were deep enough to overcome thermal motion. Dimeric structure of large molecules (such as in antibodies) contributes to the significance of this effect (Urbanija et al. 2007, 2008b).

Having observed the mediating effect of beta-2-glycoprotein I, antiphospholipid antibodies and blood plasma in phospholipid vesicles (Urbanija et al. 2007; Frank et al. 2008), it was suggested that a similar effect would take place in cells (Janša et al. 2008). However, there is an important issue that should be taken into account, namely, the outer layer of the plasma membrane contains glycolipids (Boulbitch et al. 2001). The sugar coat prevents adjacent membranes to approach each other to a distance that could be subject to attractive mediated interaction. In this work we report of adhesion of buds to the mother membrane in erythrocytes (Fig. 3B,F), in platelets (Fig. 3C) and in leukocytes (Fig. 3E). We suggest that the self-adhesion of nano-sized buds could occur if the membrane around the neck becomes depleted or nude with respect to the sugar coat, and if the appropriate mediating molecules are present in the solution. The favourable composition of membrane in the neck is attained by curvature-sorting of the membrane constituents (Gozdz and Gompper 1989, 1999; Yaghmur et al. 2007; Kralj-Iglič and Veranič 2007; Shlomovitz et al. 2011). Glycolipids with extensive parts sticking from the outer membrane layer will not likely accumulate in strongly negatively and anisotropically curved region of the neck, which enables the suggested process to take place. It can be interpreted that the particular curvature of the neck provides the field for appropriate sorting of membrane constituents in the neck.

The adhesion of the bud to the mother membrane would, however, take place only if the neck connecting the compartments were an energetically favourable structure. It was therefore of interest to study the stability of the neck(s). We have observed it indirectly, by studying the development of thermal fluctuations of a mother GPV while the necks were formed in a process of integration of a myelin-like protrusion into the mother GPV. In phospholipid systems, the existence of network of nanotubes was indicated in an 

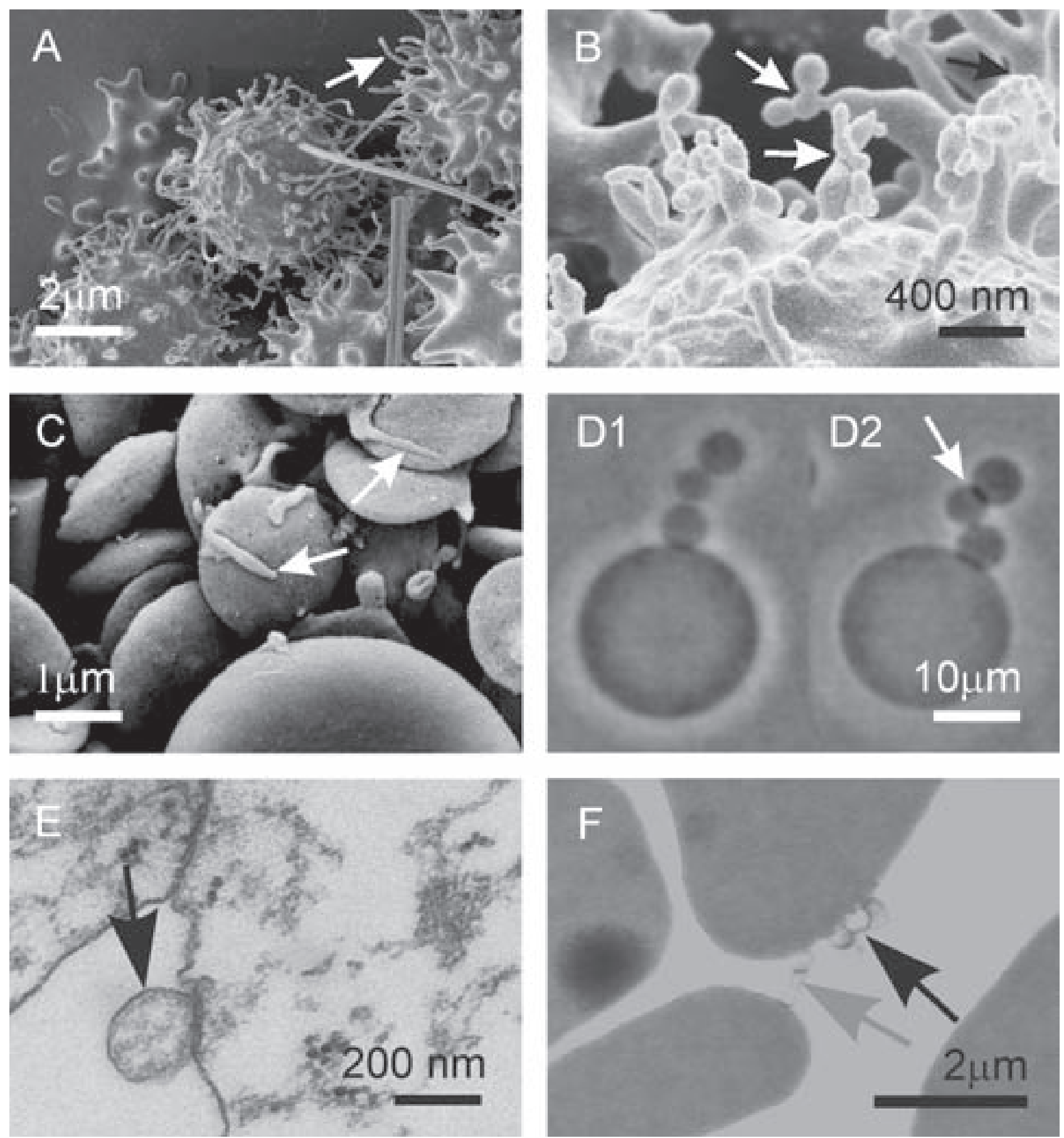

Figure 3. Budding of biological membranes and adhesion of buds to the mother membrane. A SEM image of echinocytes with tubular protrusions induced by calcium ionophore A21387 (A), a SEM image of the adhesion of beads (B), a SEM image of the adhesion of tubular platelet protrusion to the mother platelet and to the adjacent platelet (C), a phase contrast microscope image of a giant phospholipid vesicle in a suspension with added PBS-dissolved beta 2 glycoprotein I 10 minutes after the addition of the sample (D1) and 20 minutes after the addition of the sample (D2), a TEM image of a bud adhered to the leukocyte membrane (E) and a TEM image of vesicles adhered to an erythrocyte membrane (F). Arrows in panels A-C point to buds, arrow in panel $\mathrm{D}$ points to the area of adhesion between membrane parts connected by a thin neck, black arrows in $\mathrm{E}$ and $\mathrm{F}$ point to globular membranous nanostructures adhered to the plasma membrane and gray arrow in F points to a connection between cells formed by adhered nano-sized protrusions. 
experiment (Mathivet et al. 1996) which showed rapid transport of fluorescent label within the membrane between the GPVs prepared by electroformation (Angelova et al. 1992). The existence of the nanotubular network was then proved by an experiment in which the remnants of the network in the form of tubular protrusions (that are attached to the mother globule) became visible under the phase contrast microscope after undergoing a slow spontaneous shape transformation in which the average mean curvature of the vesicle decreased causing the protrusion to become shorter and thicker (Kralj-Iglič et al. 2001). Thin necks between the "beads" that are of interest in this work were formed in the last stages of this process.

Since the contour of the mother vesicle is not in the same focus plane as the protrusion it is not possible to record both at the same time. However, the peculiar stepwise pattern of the time-course of the effective radius and of the Fourier coefficients of the mother vesicle shape is in agreement with previous observations of the width of the protrusion necks (Božič et al. 2002). The necks connecting

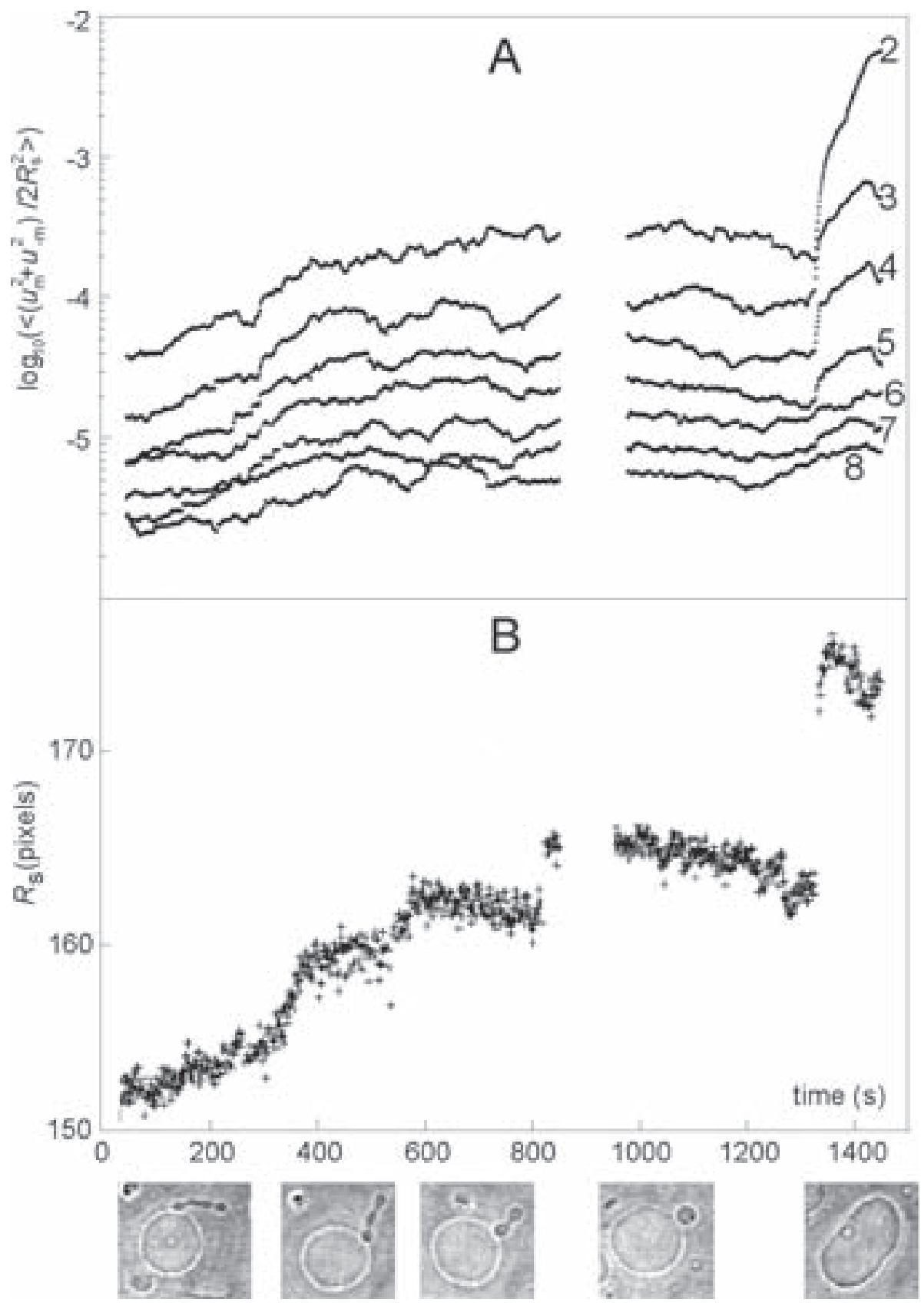

Figure 4. A. Time dependence of the average (moving averages over 100 points) square of the Fourier coefficients normalized by the square of the effective radius $\left\langle\left(u_{\mathrm{m}}+u_{-\mathrm{m}}\right)^{2} / 2 R_{\mathrm{s}}{ }^{2}\right\rangle$ for $\mathrm{m}=2$ to 8 ; the scale on the ordinate is logarithmic. B. Time dependence of the effective radius of the mother globule $R_{\mathrm{S}}$ along the same sequence where the shortened and undulated protrusion integrates with the globular part. Each point corresponds to one image. 
four beads were wider than the necks connecting three beads and these were wider than the neck that connects a single bead to the mother vesicle (Božič et al. 2002). The narrower the neck, the longer the persistence of the given number of beads (Fig. 4B). It is therefore concluded that the narrow neck tends to stabilize the shape. This effect is not limited to the neck that connects the protrusion with the mother globule but is also present in the shapes with protrusions with two or three (wider) necks, although it is not so strong.

Within the last two "steps" (times between 650-800 s and $800-1300 \mathrm{~s}$ in Fig. 4) the effective radius of the mother globule decreased (Fig. 4B). However, the effective radius decreased also after the protrusion was completely incorporated into the mother globule (times larger than $1300 \mathrm{~s}$ ). It can be expected that the fluctuations immediately after the integration of a larger amount of material into the globular part were not spherically symmetric as the inflow appeared at a certain place where the protrusion was joined with the globular part. After some time the spherically symmetric mode was more or less restored. There may be other reasons for the decrease of $R_{\mathrm{S}}$ such as the flow of the membrane and of the contents to and from the protrusion, rearrangement of the phospholipid molecules within the membrane etc. At this point the observed decrease of $R_{\mathrm{S}}$ within a "step" remains unexplained.

As the bending energy of the lowest harmonic modes of a flaccid membrane is comparable to the thermal energy, the vesicle shape spontaneously fluctuates. To eliminate the effect of these thermal fluctuations the observed coefficients $u_{m}$ were averaged over consecutive points. The moving average was used meaning that each point was calculated as the average over the interval of chosen number of points centered at the given point. Averaging over time however blurs the stepwise time dependence of Fourier coefficients. The number of points (100) was chosen as an optimal choice for both effects.

The shape transformation of the stearoyl-oleoyl-phosphatidylcholine vesicles undergoing a budding transition due to heating was analyzed (Döbereiner et al. 1995). It was found that the vesicles change abruptly as temperature $T$ is raised, from a prolate ellipsoidal shape to a shape composed of a spherical mother vesicle and a spherical daughter vesicle connected by a narrow neck (such as in Fig. 1F). A similar process was observed in dimirystoyl phosphatidyl choline vesicles by Käs and Sackmann (1991). It was also observed that in the reverse process in which the vesicles were cooled the neck opened at lower temperature indicating hysteresis (Käs and Sackmann 1991). The acknowledged theory of membrane isotropic elasticity (Miao et al. 1994) does not offer an explanation for this feature (Döbereiner et al. 1995), but the budding transition was interpreted as a first order transition (Käs and Sackmann 1991) preceded by large ther- mal shape fluctuations of a metastable state near its spinodal instability and quasi-critical slowing down (Döbereiner et al. 1995).

Also in our experiments, before opening of the neck connecting the globular part and the spherical daughter vesicle, oscillations of the neck width on the timescale of a minute were observed, indicating that the vicinity of a shape phase transition reflects a phase transition within the bilayer membrane. We suggest that this phase transition could be based on in-plane orientational ordering of phospholipid molecules.

A theory was proposed that anisotropic inclusions within the phospholipid bilayer membrane may become in-plane orientationally ordered at those regions which exhibit strongly different principal curvatures. The free energy of the equilibrium vesicle shapes that are continuously transformed from a prolate shape to the pear shape and further to the shape with a spherical protrusion connected to the mother vesicle by a thin neck exhibits a deep minimum corresponding to the shape in which the mother globule and the daughter vesicle are connected by a thin, but finite neck (Kralj-Iglič et al. 1999). In the neck, the inclusions exhibit orientational ordering which causes a decrease of the free energy (Kralj-Iglič et al. 1999).

Due to various reasons (e.g. equilibration of osmotic pressure, presence in the solution of molecules with particular properties, preferential intercalation of molecules into one of the two layers), the shape of the GPV may change. This change can be such that in some area(s) (e.g. necks) the curvature may become stronger and anisotropic. In order to constitute the membrane at that region, a phospholipid molecule may undergo a conformational change so that the shape of the molecule becomes strongly anisotropic (in the sense that not all in-plane orientations are energetically equivalent). Such molecule may be considered as a seed for an anisotropic inclusion. If the curvature relaxes, the conformational change relaxes too. We may say that such inclusion is transient. However, if the vesicle fluctuates around the shape with an anisotropic region (e.g. neck), the phospholipid molecule spends more time in a highly anisotropic state. Due to the interaction between the phospholipid molecules, clusters of highly anisotropic molecules may be formed which in turn constitute the membrane and impose the local curvature (Boulbitch 1997). Inclusions become orientationally ordered while the formation of the neck is promoted. The observed critical fluctuations may therefore indicate the vicinity of the phase transition in which a pool of the phospholipid molecules that are strongly anisotropic and orientationally ordered is localized around the narrow but finite neck. The change of the average mean curvature (presumably due to the change of the number of the molecules in the outer membrane layer) is, however, important as to drive the shape over the prolate-pear transition where the probability of the proposed mechanism becomes high. 
Theoretical models have shown that energetically favourable orientational ordering of water molecules in the vicinity of the membranes (May et al. 2008; Iglič et al. 2010; Gongadze et al. 2011; Gongadze and Iglič 2012, Velikonja et al. 2013) can importantly influence mediated interaction through a considerable decrease of the permittivity of the solution in the vicinity of the membrane. This enhances the electric field and its gradient in the close vicinity of the membrane and promotes the attractive interaction mediated by solutes with spatially distributed charge.

\section{Conclusion}

Adhesion of the membrane bud to the mother membrane due to attractive mediated interaction is a possible mechanism that could underlay anticoagulant and anti-metastatic role of molecules in the solution in contact with the cell. Our results indicate that suppression of nanovesiculation (which was observed in erythrocytes, leukocytes and platelets) involves a development of a stable (energetically favourable) neck and adhesion of the membranes of the bud and of the mother membrane to each other. Both, the stabilization of the neck and the attractive mediated interaction between membranes can be explained by orientational ordering of the system constituents.

Acknowledgements. The authors acknowledge support from the Slovenian Research Agency (projects J1-4109 and J3-4108), EUREKA grant IMIPEB, and Novartis International AG. This work is subject to no conflict of interest.

\section{References}

Abramowitz M., Stegun I. A. (1970): Handbook of Mathematical Functions. 9th edition, Dover Publications Inc.

Al-Nedawi K., Meehan B., Micallef J., Lhotak V., May L., Guha A., Rak J. (2008): Intercellular transfer of the oncogenic EGFRvIII via tumor cell derived microvesicles. Nat. Cell Biol. 10, 619-624 http://dx.doi.org/10.1038/ncb1725

Ambrožič A., Čučnik S., Tomšič N., Urbanija J., Lokar M., Babnik B., Rozman B., Iglič A., Kralj-Iglič V. (2006) Interaction of giant phospholipid vesicles containing cardiolipin and cholesterol with $\beta$-glycoprotein-I and anti- $\beta$-glycoprotein-I antibodies. Autoimmun. Rev. 6, 10-15 http://dx.doi.org/10.1016/j.autrev.2006.03.001

Angelova M. I., Soléau S., Méléard Ph., Faucon F., Bothorel P. (1992): Preparation of giant vesicles by external AC electric fields. Kinetics and applications. Prog. Coll. Pol. Sci. 89, 127-131 http://dx.doi.org/10.1007/BFb0116295

Bagatolli L. A., Parasassi T., Gratton E. (2000): Giant phospholipid vesicles: comparison among the whole lipid sample characteristics using different preparation methods: A two photon fluorescence microscopy study. Chem. Phys. Lipids 105, 135-147
http://dx.doi.org/10.1016/S0009-3084(00)00118-3

Bivas I., Hanusse P., Bothorel P., Lalanne J., Aguerre-Chariol O. (1987): An application of the optical microscopy to the determination of the curvature elastic modulus of biological and model membranes. J. Physique 48, 855-867 http://dx.doi.org/10.1051/jphys:01987004805085500

Bivas I., Bivolarski L., Mitov M. D., Derzhanski A. (1992): Correlations between the form fluctuation modes of flaccid quasispherical lipid vesicles and their role in the calculation of the curvature elastic modulus of the vesicle membrane - numerical results. J. Phys. II 2, 1423-1438 http://dx.doi.org/10.1051/jp2:1992210

Bivas I., Méléard P. (2003): Bending elasticity and bending fluctuations of lipid bilayer containing an additive. Phys. Rev. E 67, 012901 http://dx.doi.org/10.1103/PhysRevE.67.012901

Bivas I. (2010): Shape fluctuations of nearly spherical lipid vesicles and emulsion droplets. Phys. Rev. E 81, 061911 http://dx.doi.org/10.1103/PhysRevE.81.061911

Boulbitch A., Guttenberg Z., Sackmann E. (2001): Kinetics of membrane adhesion mediated by ligand-receptor interaction studied with a biomimetic system. Biophys. J. 81, 2743-2751 http://dx.doi.org/10.1016/S0006-3495(01)75917-6

Boulbitch A. A. (1997): Crystallization of proteins accompanied by formation of a cylindrical surface. Phys. Rev. E 56, 3395-3400

http://dx.doi.org/10.1103/PhysRevE.56.3395

Bouma B., De Groot P. G., van den Elsen J. M., Ravelli R. B., Schouten A., Simmelink M. J., Derksen R. H., Kroon J., Gros P. (1999): Adhesion mechanism of human beta(2)-glycoprotein I to phospholipids based on its crystal structure. EMBO J. 18, 5166-5174

http://dx.doi.org/10.1093/emboj/18.19.5166

Božič B., Gomišček G., Kralj-Iglič V., Svetina S., Žekš B. (2002): Shapes of phospholipid vesicles with beadlike protrusions. Eur. Biophys. J. 31, 487-496 http://dx.doi.org/10.1007/s00249-002-0247-x

Dignat-George, F., Camoin-Jau, L., Sabatier, F., Arnoux D., Anfosso F., Bardin N., Veit V., Combes V., Gentile S., Moal V. (2004): Endothelial microparticles: a potential contribution to the thrombotic complications of the antiphospholipid syndrome. Thromb. Haemostasis 91, 667-673

Distler J. H. W., Pisetsky D. S., Huber L. C., Kalden J. R., Distler O. (2005): Microparticles as regulators of inflammation: novel players of cellular crosstalk in the rheumatic diseases. Arthritis Rheum. 52, 3337-3348 http://dx.doi.org/10.1002/art.21350

Döbereiner H. G., Evans E., Seifert U., Wortis M. (1995): Spinodal fluctuations of budding vesicles. Phys. Rev. Lett. 75, 3360 http://dx.doi.org/10.1103/PhysRevLett.75.3360

Faucon J. F., Mitov M. D., Méléard P., Bivas I., Bothorel P. (1989): Bending elasticity and thermal fluctuations of lipid-membranes - theoretical and experimental requirements. J. Phys. 50, 2389-2414 http://dx.doi.org/10.1051/jphys:0198900500170238900

Fevrier B., Vilette D., Archer F., Loew D., Faigle W., Vidal M., Laude H., Raposo G. (2004): Cells release prions in association with exosomes. Proc. Natl. Acad. Sci. U.S.A. 101, 9683-9688 http://dx.doi.org/10.1073/pnas.0308413101 
Frank M., Manček-Keber M., Kržan M., Sodin-Šemrl S., Jerala R., Iglič A., Rozman B., Kralj-Iglič V. (2008): Prevention of microvesiculation by adhesion of buds to the mother cell membrane - a possible anticoagulant effect of healthy donor plasma. Autoimmun. Rev. 7, 240-245 http://dx.doi.org/10.1016/j.autrev.2007.11.015

Galkina S. I., Molotkovsky J. G., Ullrich V., Sudina G. F. (2005): Scanning electron microscopy study of neutrophil membrane tubulovesicular extensions (cytonemes) and their role in anchoring, aggregation and phagocytosis. The effect of nitric oxide. Exp. Cell Res. 304, 620-629 http://dx.doi.org/10.1016/j.yexcr.2004.12.005

Genova J., Zheliaskova A., Vitkova V., Mitov M. D. (2009): Stroboscopic illumination study of the dynamics of fluctuating vesicles. J. Optoelectron. Adv. M. 11, 1222-1225

Gimsa U., Iglič A., Fiedler S., Zwanzig M., Kralj-Iglič V., Jonas L., Gimsa J. (2007): Actin is not required for nanotubular protrusions of primary astrocytes grown on metal nano-lawn. Mol. Membr. Biol. 24, 243-255

http://dx.doi.org/10.1080/09687860601141730

Gongadze E., van Rienen U., Kralj-Iglič V., Iglič A. (2011): Langevin Poisson-Boltzmann equation: point-like ions and water dipoles near charged membrane surface. Gen. Physiol. Biophys. 30, $130-137$ http://dx.doi.org/10.4149/gpb_2011_02_130

Gongadze E., Iglič A. (2012): Decrease of permittivity of an electrolyte solution near a charged surface due to saturation and excluded volume effects. Bioelectrochemistry 87, 199-203 http://dx.doi.org/10.1016/j.bioelechem.2011.12.001

Gonzalez R. C., Woods R. E. (1992): Digital Image Processing, Addison-Wesley, Reading, Boston

Gozdz W. T., Gompper G. (1998): Composition-driven shape transformations of membranes of complex topology. Phys. Rev. Lett. 80, 4213-4216 http://dx.doi.org/10.1103/PhysRevLett.80.4213

Gozdz W. T., Gompper G. (1999): Shapes and shape transformations of two-component membranes of complex topology. Phys. Rev. E 59, 4305-4316 http://dx.doi.org/10.1103/PhysRevE.59.4305

Hägerstrand H., Isomaa B. (1989): Vesiculation induced by amphiphiles in erythrocytes. Biochim. Biophys. Acta 982, 179-186 http://dx.doi.org/10.1016/0005-2736(89)90053-9

Hägerstrand H., Isomaa B. (1992): Morphological characterization of exovesicles and endovesicles released from human erythrocytes following treatment with amphiphiles. Biochim. Biophys. Acta 1109, 117-126 http://dx.doi.org/10.1016/0005-2736(92)90074-V

Iglič A., Hägerstrand H., Bobrowska-Hägerstrand M., Arrigler V., Kralj-Iglič V. (2003): Possible role of phospholipid nanotubes in directed transport of membrane vesicles. Phys. Lett. 310, 493-497 http://dx.doi.org/10.1016/S0375-9601(03)00449-3

Iglič A., Babnik B., Bohinc K., Fošnarič M., Hägerstrand H., Kralj-Iglič V. (2007): On the role of anisotropy of membrane constituents in formation of a membrane neck during budding of a multicomponent membrane. J. Biomech. 40, $579-585$

http://dx.doi.org/10.1016/j.jbiomech.2006.02.006
Iglič A., Gongadze E., Bohinc K. (2010): Excluded volume effect and orientational ordering near charged surface in solution of ions and Langevin dipoles. Bioelectrochemistry 79, 223-227 http://dx.doi.org/10.1016/j.bioelechem.2010.05.003

Janowska-Wieczorek A., Marquez-Curtis L. A., Wysoczynski M., Ratajczak M. Z. (2006): Enhancing effect of platelet-derived microvesicles on the invasive potential of breast cancer cells. Transfusion 46, 1199-1209 http://dx.doi.org/10.1111/j.1537-2995.2006.00871.x

Janša R., Šuštar V., Frank M., Sušanj P., Bešter J., Manček-Keber M., Kržan M., Iglič A. (2008): Number of microvesicles in peripheral blood and ability of plasma to induce adhesion between phospholipid membranes in 19 patients with gastrointestinal diseases. Blood Cells Mol. Dis. 41, 124-132 http://dx.doi.org/10.1016/j.bcmd.2008.01.009

Jorgačevski J., Fošnarič M., Vardjan N., Stenovec M., Potokar M., Kreft M., Kralj-Iglič V., Iglič A., Zorec R. (2010): Fusion pore stability of peptidergic vesicles. Mol. Membr. Biol. 27, $65-80$ http://dx.doi.org/10.3109/09687681003597104

Junkar I., Šuštar V., Frank M., Janša V., Bedina Zavec A., Rozman B., Mozetič M., Hägerstrand H., Kralj-Iglič V. (2009): Blood and synovial microparticles as revealed by atomic force and scanning electron microscope. Open Autoimmun J. 1, e50-58 http://dx.doi.org/10.2174/1876894600901010050

Käs J., Sackmann E. (1991): Shape transitions and shape stability of giant phospholipid vesicles in pure water induced by areato-volume changes. Biophys. J. 60, 825-844 http://dx.doi.org/10.1016/S0006-3495(91)82117-8

Kertesz Z., Yu B. B., Steinkasserer A., Haupt H., Benham A., Sim R. B. (1995): Characterization of binding of human beta 2-glycoprotein I to cardiolipin. Biochem. J. 310, 315-321

Kralj-Iglič V., Heinrich V., Svetina S., Žekš B. (1999): Free energy of closed membrane with anisotropic inclusions. Eur. Phys. J. B 10, 5-8 http://dx.doi.org/10.1007/s100510050822

Kralj-Iglič V., Iglič A., Hägerstrand H., Peterlin P. (2000): Stable tubular microexovesicles of the erythrocyte membrane induced by dimeric amphiphiles. Phys. Rev. E 61, 4230-4234 http://dx.doi.org/10.1103/PhysRevE.61.4230

Kralj-Iglič V., Gomišček G., Majhenc J., Arrigler V., Svetina S. (2001): Myelin-like protrusions of giant phospholipid vesicles prepared by electroformation. Colloids Surf. A 181, 315 http://dx.doi.org/10.1016/S0927-7757(00)00802-5

Kralj-Iglič V., Iglič A., Gomišček G., Sevšek F., Arrigler V., Hägerstrand H. (2002): Microtubes and nanotubes of a phospholipid bilayer membrane. J. Phys. A: Math. Gen. 35, 1533-1549 http://dx.doi.org/10.1088/0305-4470/35/7/305

Kralj-Iglič V., Babnik B., Gauger D. R., May S., Iglič A. (2006): Quadrupolar ordering of phospholipid molecules in narrow necks of phospholipid vesicles. J. Stat. Phys. 125, 727-752 http://dx.doi.org/10.1007/s10955-006-9051-9

Kralj-Iglič V., Veranič P. (2007): Curvature-induced sorting of bilayer membrane constituents and formation of membrane rafts. In: Advances in Planar Lipid Bilayers and Liposomes. (Ed. A. Leitmanova Liu), pp. 129-149, Elsevier, Amsterdam, London 
Kralj-Iglič V. (2012): Stability of membranous nanostructures - a possible key mechanism in cancer progression. Int. J. Nanomedicine 7, 3579-3596 http://dx.doi.org/10.2147/IJN.S29076

Likar B., Maintz J. B. A., Viergever M. A., Pernuš F. (2000): Retrospective shading correction based on entropy minimization, J. Microscopy 197, 285-295 http://dx.doi.org/10.1046/j.1365-2818.2000.00669.x

Likar B., Viergever M. A., Pernuš F. (2001): Retrospective correction of MR intensity inhomogeneity by information minimization. IEEE T. Med. Imag. 20, 1398-1410 http://dx.doi.org/10.1109/42.974934

Mareš T., Daniel M., Perutkova Š., Perne A., Dolinar G., Iglič A. Rappolt M., Kralj-Iglič V. (2008): Role of phospholipid asymmetry in the stability of inverted hexagonal mesoscopic phases. J. Phys. Chem. B 8, 16575-16584 http://dx.doi.org/10.1021/jp805715r

Mathivet L., Cribier S., Devaux P. F. (1996): Shape change and physical properties of giant phospholipid vesicles prepared in the presence of an AC electric field. Biophys. J. 7, 1112-1121 http://dx.doi.org/10.1016/S0006-3495(96)79693-5

May S., Iglič A., Reščič J., Maset S., Bohinc K. (2008): Bridging like-charged macroions through long divalent rod-like ions. J. Phys. Chem. B 112, 1685-1692 http://dx.doi.org/10.1021/jp073355e

Méléard P., Gerbeaud C., Pott T., Fernandez-Puente L., Bivas I. Mitov M. D., Dufourcq J., Bothorel P. (1997): Bending elasticities of model membranes: Influences of temperature and sterol content. Biophys. J. 72, 2616-2629 http://dx.doi.org/10.1016/S0006-3495(97)78905-7

Miao L., Seifert U., Wortis M., Döbereiner H. G. (1994): Budding transitions of fluid-bilayer vesicles: the effect of area-difference elasticity. Phys. Rev. E 49, 5389-5407 http://dx.doi.org/10.1103/PhysRevE.49.5389

Mrvar-Brečko A., Šuštar V., Janša V., Štukelj R., Janša R., Mujagić E., Kruljc P., Iglič A., Hägerstrand H., Kralj-Iglič V. (2010): Isolated microvesicles from peripheral blood and body fluids as observed by scanning electron microscope. Blood Cells Mol. Dis. 44, 307-312 http://dx.doi.org/10.1016/j.bcmd.2010.02.003

Müller I., Klocke A., Alex M., Kotzsch M., Luther T., Morgenstern E., Zieseniss S., Zahler S., Preissner K., Engelmann B. (2000): Intravascular tissue factor initiates coagulation via circulating microvesicles and platelets. FASEB J. 17, 476-478

Pavlič J. I., Genova J., Zheliaskova A., Iglič A., Mitov M. D. (2010): Electroformation of neutral and negatively charged phospholipid giant vesicles under physiological conditions. CR. Acad. Bulg. Sci. 63, 497-502

Peterlin P., Arrigler V. (2008): Electroformation in a flow chamber with solution exchange as a means of preparation of flaccid giant vesicles. Colloids Surf. B 64, 77-87 http://dx.doi.org/10.1016/j.colsurfb.2008.01.004

Pisetsky D. S. (2009): Microparticles as biomarkers in autoimmunity: from dust bin to center stage. Arthritis Res. Ther. 11, 135 http://dx.doi.org/10.1186/ar2856

Press W. H., Flannery B. P., Teukolsky S. A., Wetterling W. T. (1992): Numerical Recipes. Example Book (C++). 2nd ed., Cambridge University Press, Cambridge
Rak J. (2010): Microparticles in cancer. Semin. Thromb. Hemost. 36, 888-906 http://dx.doi.org/10.1055/s-0030-1267043

Rappolt M., Hodzic A., Sartori B., Ollivon M., Laggner P. (2008): Conformational and hydrational properties during the Lb- to La- and La- to HII-phase transition in phosphatidylethanolamine. Chem. Phys. Lipids 154, 46-55 http://dx.doi.org/10.1016/j.chemphyslip.2008.02.006

Ratajczak J., Wysoczynski M., Hayek F., Janowska-Wieczorek A., Ratajczak M. Z. (2006): Membrane-derived microvesicles: important and underappreciated mediators of cell to cell communication. Leukemia 20, 1487-1495 http://dx.doi.org/10.1038/sj.leu.2404296

Robertson C., Booth S. A., Beniac D. R., Coulthart M. B., Booth T. F., McNicol A. (2006): Cellular prion protein is released on exosomes from activated platelets. Blood 107, 3907-3911 http://dx.doi.org/10.1182/blood-2005-02-0802

Russ J. C. (1995): The Image Processing Handbook. 2nd ed., IEEE Press, Boca Raton

Rustom A., Saffrich R., Markovic I., Walther P., Gerdes H. H. (2004): Nanotubular highways for intercellular organelle transport. Science 303, 1007-1010 http://dx.doi.org/10.1126/science.1093133

Schara K., Janša V., Šuštar V., Dolinar D., Pavlič J. I., Lokar M., Kralj-Iglič V., Veranič P., Iglič A. (2009): Mechanisms for the formation of membranous nanostructures in cell-to-cell communication. Cell. Mol. Biol. Lett. 14, 636-656 http://dx.doi.org/10.2478/s11658-009-0018-0

Sevšek F., Gomišček G. (2004): Shape determination of attached fluctuating phospholipid vesicles. Comput. Meth. Prog. Bio. 73, 189-194

http://dx.doi.org/10.1016/S0169-2607(03)00065-8

Shlomovitz R., Gov N. S., Roux A. (2011): Membrane-mediated interactions and the dynamics of dynamin oligomers on membrane tubes. New J. Phys. 13, 065008 http://dx.doi.org/10.1088/1367-2630/13/6/065008

Šuštar V., Janša R., Frank M., Hägerstrand H., Kržan M., Iglič A., Kralj-Iglič V. (2009): Suppression of membrane microvesiculation - A possible anticoagulant and anti-tumor progression effect of heparin. Blood Cells Mol. Dis. 42, 223-227 http://dx.doi.org/10.1016/j.bcmd.2009.01.012

Šuštar V., Bedina-Zavec A., Štukelj R., Frank M., Bobojević G., Janša R., Ogorevc E., Kruljc P., Mam K., Šimunič B. (2011a): Nanoparticles isolated from blood: a reflection of vesiculability of blood cells during the isolation process. Int. J. Nanomed. 6, 2737-2748

Šuštar V., Bedina-Zavec A., Štukelj R., Frank M., Ogorevc E., Janša R., Mam K., Veranič P., Kralj-Iglič V. (2011b): Post-prandial rise of microvesicles in peripheral blood of healthy human donors. Lipids Health Dis. 10, 47 http://dx.doi.org/10.1186/1476-511X-10-47

Šuštar V., Zelko J., Lopalco P., Lobasso S., Otta A., Poklar Ulrih N., Corcelli A., Kralj-Iglič V. (2012): Morphology, biophysical properties and protein-mediated fusion of archaeosomes. PLoS ONE, 7, e39401 http://dx.doi.org/10.1371/journal.pone.0039401

Urbanija J., Tomšič N., Lokar M., Ambrožič A., Čučnik S., Rozman B., Kandušer M., Iglič A., Kralj-Iglič V. (2007): Coalescence of phospholipid membranes as a possible ori- 
gin of anticoagulant effect of serum proteins. Chem. Phys. Lipids, 150, 49-57 http://dx.doi.org/10.1016/j.chemphyslip.2007.06.216

Urbanija J., Babnik B., Frank M., Tomšič N., Rozman B., KraljIglič V., Iglič A. (2008a): Attachment of ß2-glycoprotein I to negatively charged liposomes may prevent the release of daughter vesicles from the parent membrane. Eur. Biophys. J. 37, 1085-1095 http://dx.doi.org/10.1007/s00249-007-0252-1

Urbanija J., Bohinc K., Bellen A., Maset S., Iglič A., Kralj-Iglič V., Kumar P.B. (2008b): Attraction between negatively charged surfaces mediated by spherical counterions with quadrupolar charge distribution. J. Chem. Phys. 129, 105101 http://dx.doi.org/10.1063/1.2972980

Usenik P., Vrtovec T., Pernuš F. (2011): Automated tracking and analysis of phospholipid vesicle contours in phase contrast microscopy images. Med. Biol. Eng. Comput. 49, 957-966 http://dx.doi.org/10.1007/s11517-011-0789-0

Vella L. J., Greenwood D. L. V., Cappai R., Scheerlinck J. F., Hill A. F. (2008): Enrichment of prion protein in exosomes derived from ovine cerebral spinal fluid. Vet. Immunol. Immunop. 124, 385-393 http://dx.doi.org/10.1016/j.vetimm.2008.04.002

Velikonja A., Perutkova Š., Gongadze E., Kramar P., Polak A., Maček-Lebar A., Iglič A. (2013): Monovalent ions and water dipoles in contact with dipolar zwitterionic lipid headroups theory and MD simulations, Int. J. Mol. Sci. 14, 2846-2861 http://dx.doi.org/10.3390/ijms14022846

Veranič P., Lokar M., Schuetz G. J., Weghuber J., Wieser S., Hägerstrand H., Kralj-Iglič V., Iglič A. (2008): Different types of cell-to-cell connections mediated by nanotubular structures. Biophys. J. 95, 4416-4425 http://dx.doi.org/10.1529/biophysj.108.131375

Vitkova V., Genova J., Méléard P. (2003): Influence of alamethicin on the passive water permeability of model lipid membranes and on the morphology of giant lipid vesicles. J. Mat. Sci. - Matter. El. 14, 819-820 http://dx.doi.org/10.1023/A:1026129910215

Wang S. X., Cai G. P., Sui S. F. (1998): The insertion of human apolipoprotein $\mathrm{H}$ into phospholipid membranes: a monolayer study. Biochem J. 335, 225-232

Yaghmur A., Laggner P., Zhang S., Rappolt M. (2007): Tuning curvature and stability of monoolein bilayers by designer lipid-like peptide surfactants. PLoS ONE 2, e479 http://dx.doi.org/10.1371/journal.pone.0000479

Yu J. L., May L., Lhotak V., Shahrzad S., Shirasawa S., Weitz J. I., Coomber B. L., Mackman N., Rak J. W. (2005): Oncogenic events regulate tissue factor expression in colorectal cancer cells: Implications for tumor progression and angiogenesis. Blood 105, 1734-1741 http://dx.doi.org/10.1182/blood-2004-05-2042

Received: June 30, 2012

Final version accepted: July 31, 2012 